\title{
DISTRIBUTION OF DUST IN THE ORION NEBULA
}

\author{
LINDSEY F. SMITH \\ Max-Planck-Institut für Radioastronomie, Bonn, F.R.G.
}

\begin{abstract}
The distribution of the dust is found by comparing 3-cm brightness temperatures, $\mathrm{H} \alpha$ surface brightness, and $\mathrm{H} \beta$ surface brightness. The dust is probably mixed with the gas. The Balmer radiation originates in front of the dark bay.
\end{abstract}

Comparison is made between 3 -cm brightness temperatures determined by Wink (1973) with the NRAO aperture synthesis interferometer and $\mathrm{H} \alpha$ and $\mathrm{H} \beta$ surface brightnesses determined from narrow band interference photographs obtained in conjunction with the Marseille group with the Haute Province Observatory 193-cm telescope.

The $\mathrm{H} \alpha$ intensity is $\sim 1 / 5$ that expected at the positions of peak radio brightness and $\sim 1 / 2$ that expected in the neighbouring regions. Yet the $\mathrm{H} \alpha / \mathrm{H} \beta$ ratio is approximately constant over the whole central region. This implies that the dust is mixed with the gas rather than being a foreground phenomenon.

The intensity of the Balmer radiation from the darkest parts of the dark bay is $\sim 1 / 5$ of that of adjacent regions, but the $\mathrm{H} \alpha / \mathrm{H} \beta$ ratio is not greater than in adjacent regions. Radio observations with resolution as high as $2^{\prime}$ (Schraml and Mezger, 1969) have not detected any structure in the region of the bay. This implies that most of the observed Balmer radiation originates in front of the bay. The gas in the bay must be neutral and the extinction by the dust must be high enough to prevent significant contribution to the observed Balmer radiation from regions behind the bay.

\section{References}

Schraml, J. and Mezger, P. G.: 1969, Astrophys. J. 156, 269.

Wink, J.: 1973, private communication.

Lindsey F. Smith

Max-Planck-Institut für Radioastronomie,

Auf dem Hügel 69,

53 Bonn 1, F.R.G.

\section{DISCUSSION}

Habing: To what extent do your results agree with those of Münch and Persson?

L. F. Smith: Since Münch and Persson have not used radio observations, I have not studied the paper carefully enough to answer the question.

Van Woerden: Do I understand that you observe $\mathrm{H} \alpha$ and $\mathrm{H} \beta$ in front of the dark bay? If so, how can they be reddened? 
L. F. Smith : Reddening would then be due only to dust mixed with the emitting gas.

Zuckerman: Could half the reddening be due to foreground material?

L. F. Smith: Only if the foreground material covered just the central part of the nebula.

Greenberg: In estimating the ratio of $\mathrm{H} \alpha$ to $\mathrm{H} \beta$ would an anomolous extinction curve characterized by different total to selective extinction strongly modify your physical picture?

L.F. Smith: The ratio of 1.5 that I assumed between $\mathrm{H} \alpha$ and $\mathrm{H} \beta$ optical depths is for the Whitford reddening curve with $R=A_{V} / E_{B-V}=3$. It is possible in Orion that $R$ is greater than 3. While it would not affect the simple conclusion made here that most of the dust is inside the nebula, the possibility must be allowed for in the final reduction.

Baars: Now that you have these beautiful high angular resolution data in radio and $\mathrm{H} \alpha, \mathrm{H} \beta$ wavelengths, are you able to map the distribution of the dust by your method?

L.F. Smith: That is the aim of the program. For this initial report I have only calculated the numbers at key points.

Pishmish : A qualitative confirmation for the existence of dust in the Orion region is provided by the Fabry-Pérot interferograms which we have obtained on that region. These show that in between the interference rings of $\mathrm{H} \alpha$ there is emission due to the continuum. This continuum may arise from the re-emission by the dust of the radiation from the embedded Trapezium stars. The interferograms may also help in mapping the dust distribution in regions where the continuum emission is observable. 